\title{
Análisis del impacto del confinamiento en los resultados de evaluación de una asignatura del grado de Ingeniería Informática*
}

\author{
Carlos T. Calafate ${ }^{1}$, José M. Cecilia ${ }^{1}$ y Juan-Carlos Cano ${ }^{1}$ \\ ${ }^{1}$ Escuela Técnica Superior de Ingeniería Informática (ETSINF), Universitat Politècnica de \\ València (UPV)
}

\begin{abstract}
The sudden changes to the teaching/learning methodology derived from the COVID-19 outbreak has forced instructors to quickly adapt their standard courses to online teaching procedures in record time. As expected, though, such changes were not without their drawbacks. In this paper, we provide a study made regarding the effectiveness of the alternative evaluation procedures adopted for a the course Design and Configuration of Local Area Networks in the degree on Informatics at the Universitat Politècnica de València (UPV) in Spain. Results show that the online evaluation methods adopted, despite the efforts to mitigate plagiarism, cannot correctly mimic the face-to-face approaches. In fact, we verify a consistent bias of results generating excessively high scores. Such deficiencies in the process are partially endorsed by students' opinions as well.
\end{abstract}

Keywords: COVID-19, evaluation methodology, online teaching.

\begin{abstract}
Resumen
Los cambios repentinos en la metodología de enseñanza/aprendizaje provocados por los efectos de la pandemia del COVID-19 han obligado al profesorado universitario a adaptar rápidamente su docencia presencial a una metodología de enseñanza a distancia tiempo récord. Sin embargo, como era de esperar, estos cambios forzados no estuvieron exentos de varios inconvenientes y efectos colaterales. En este trabajo se presenta un estudio sobre la efectividad de los procedimientos alternativos de evaluación adoptados en la asignatura Diseño y Configuración de Redes de Área Local del Grado en Ingeniería Informática de la Universitat Politècnica de València (UPV), España. Los resultados muestran que la metodología de evaluación a distancia utilizada, a pesar de las herramientas utilizadas para mitigar intentos de copia, no son comparables a las
\end{abstract}

\footnotetext{
* Trabajo financiado por el Departamento de Informática de Sistemas y Computadores de la Universitat Politècnica de València.
} 
soluciones presenciales habituales. En términos de resultados, se verifica que conducen a un sesgo importante de los mismos, generando puntuaciones excesivamente altas. Estas deficiencias del proceso han sido parcialmente respaldadas por las opiniones de los estudiantes.

Keywords: COVID-19, Metodología de evaluación, Enseñanza a distancia.

\section{Introducción}

La crisis mundial del COVID-19 ha afectado a todas las áreas de la sociedad, obligando a los diferentes agentes a realizar cambios forzosos en un intento de mitigar los efectos generados por la pandemia (Nicola y col. 2020). El campo de la educación, y específicamente la educación universitaria, no ha sido excepción. Los diferentes niveles jerárquicos asociados a las universidades públicas han emitido diferentes medidas urgentes para atender las restricciones provocadas por el avance de casos de COVID-19 en diferentes países (Schleicher 2020).

Durante el segundo semestre del año académico 2019-2020, las universidades españolas han experimentado fuertes cambios para abordar el repentino desafío de cambiar las clases presenciales a una educación a distancia en pocos días. De hecho, estos cambios han provocado diferentes problemas (Odriozola-González y col. 2020; Sahu 2020) en el entorno universitario, entre los que destaca, sin lugar a dudas, los procedimientos de evaluación de las asignaturas. La adopción de evaluaciones a distancia en asignaturas que fueron previamente diseñadas para ser impartidas y, por tanto evaluadas, mediante un metodología presencial ha sido un desafío ingente (Kearns 2012). En primer lugar, el profesorado ha tenido que cambiar los procedimientos de evaluación para que se puedan realizar remotamente. Esto ha impedido garantizar la fiabilidad de la evaluación, dadas las restricciones que tiene el profesorado para detectar y evitar posibles copias, plagio, etc., es decir, todo aquello que impida la medición individual y precisa de los conocimientos de cada alumno en concreto. Además, las prácticas de laboratorio no se pueden realizar a distancia en muchos casos (Cantabella y col. 2020). Por último, pero no menos importante, los estudiantes que no disponían de una buena conexión a Internet han estado en clara desventaja con el resto de compañeros, pudiéndoles haber afectado negativamente en sus calificaciones (Alruwais, Wills y Wald 2018).

En este estudio, detallamos el procedimiento de adaptación a la modalidad a distancia de Diseño y Configuración de Redes de Área Local (DCLAN), asignatura de tercer curso del Grado de Ingeniería en Informática de la Universitat Politècnica de València (UPV), como consecuencia de las medidas sanitarias impuestas en el RD463/2020, de 14 de Marzo, por el que se declaró el estado de alarma para la gestión de la situación de crisis sanitaria de la pandemia del COVID-19. En este contexto, el objetivo central de este trabajo es realizar un análisis del impacto de las metodologías alternativas de evaluación adoptadas (derivadas del confinamiento) en términos de los resultados de evaluación de un grupo de 110 estudiantes, todos ellos de la rama de Tecnologías de la Información, y en la percepción de dichos estudiantes sobre los diferentes enfoques utilizados para tratar de mitigar el plagio. Procede destacar que dicho procedimiento de evaluación era ya considerado en (Timmis y col. 2016) como una de las principales asignaturas pendientes en la educación a distancia a nivel universitario.

Este trabajo está organizado de la siguiente manera: en la sección 2 se presentan algunos trabajos relacionados, destacando la originalidad del presente estudio. En la sección 3 se presenta brevemente la asignatura, y a continuación, en la sección 4, se detallan los cambios realizados para adaptarla a la modalidad a distancia. En la sección 5 se presentan los resultados de evaluación del alumnado 
y de las encuestas realizadas. Por último, en la sección 6, se presentan las principales conclusiones de este trabajo.

\section{Trabajos relacionados}

En la literatura reciente se pueden encontrar varios trabajos que detallan los diferentes retos que surgen en la enseñanza a distancia derivados de la pandemia del COVID-19. Mishra, Gupta y Shree (Mishra, Gupta y Shree 2020) abordaron los principales requisitos de los procedimientos de enseñanza-aprendizaje en educación a distancia, motivados por las restricciones del COVID-19. También mostraron cómo los recursos existentes en las instituciones educativas pueden transformar efectivamente la educación presencial en educación a distancia con la ayuda de clases virtuales y otras herramientas virtuales que son críticas en este panorama educativo en constante cambio. Los autores concluyeron que desarrollar enfoques multimodales para lograr los objetivos del curso es una buena estrategia de cara a abordar los retos de la educación a distancia.

Rapanta et al. (Rapanta y col. 2020) profundizaron en este contenido pedagógico relacionado con el aprendizaje a distancia. El principal objetivo de su trabajo ha sido el de ayudar a los profesores universitarios que tienen poca experiencia con dicho tipo de enseñanza. Sus hallazgos incluyeron la necesidad de diseñar actividades de aprendizaje adaptadas al nuevo contexto con una adaptación de su evaluación a estos nuevos requisitos, así como la combinación de tres tipos de presencia (social, cognitiva y facilitadora).

Brown (Brown 2020) analizó cómo las sociedades de la OCDE han mantenido la escolarización durante el COVID-19, estableciendo una base sólida para avanzar hacia una solución de red de aprendizaje. Los métodos utilizados en estos países se reflejaron en varios escenarios. Nuere y de Miguel (Nuere y Miguel 2020) mostraron una interesante comparación entre dos universidades españolas diferentes: la Universidad de La Rioja, que es una universidad puramente virtual, y la Universidad Politécnica de Madrid, que es una universidad tradicional, es decir, que los docentes se enfrentaron a una curva de aprendizaje más lenta con respecto a aquellos que estaban acostumbrados a impartir docencia a distancia. Concluyeron que esta crisis está siendo la fuerza impulsora detrás de la revolución de la enseñanza a distancia. Además, destacaron herramientas y elementos esenciales en este nuevo escenario de enseñanza-aprendizaje. En el libro, 'Enseñanza, tecnología y educación docente durante la pandemia COVID-19: historias del campo' (Ferdig y col. 2020), varios autores muestran sus estrategias pedagógicas virtuales durante la pandemia COVID-19. Bao (Bao 2020) muestra el caso de la Universidad de Pekín durante el período pandémico, con especial énfasis en el cambio hacia la enseñanza a distancia. Se presentan seis estrategias específicas para resumir las experiencias actuales de enseñanza a distancia para profesores universitarios, los cuales podrían realizar educación a distancia en circunstancias similares, con cinco principios de alto impacto para dicho tipo de enseñanza. Entre ellos podemos destacar un apoyo adecuado del profesorado y auxiliares docentes a los estudiantes, y la elaboración de un plan de contingencia para hacer frente a incidentes inesperados de las plataformas de educación virtual.

La OCDE (Schleicher 2020) publicó un libro que muestra algunos indicadores de Education at a Glance que fueron seleccionados por su particular relevancia en el contexto actual. Se centró en la respuesta de varios países de la OCDE, y en el impacto de las medidas adoptadas por ellos en la pandemia del COVID-19. También analizó varios temas, incluido la financiación público de la educación en los países de la OCDE, la movilidad internacional de estudiantes, las distracciones respecto a un entorno tradicional, las medidas para garantizar el aprendizaje de los estudiantes durante el cierre de escuelas, la preparación de los maestros para apoyar el aprendizaje digital, 
cómo y cuándo reabrir escuelas, el tamaño de las clases, los parámetros críticos para la reapertura, y la educación vocacional durante el cierre del COVID-19.

Este trabajo difiere de los anteriores porque abordamos específicamente cuestiones relacionadas con la evaluación a distancia en el periodo de la pandemia, comparando los resultados de dicha evaluación con los resultados obtenidos mediantes exámenes presenciales tradicionales.

\section{Descripción general de la asignatura}

Diseño y Configuración de Redes de Área Local (DCLAN) es una asignatura obligatoria para todos aquellos estudiantes matriculados en el Grado en Ingeniería Informática de la Universitat Politècnica de València (UPV, Valencia, España). En concreto, DCLAN se encuentra en la especialización en Tecnologías de la Información (TI) donde contribuye de forma significativa a adquirir competencias clave del grado y de la especialización, tales como: (i) Conocimiento y aplicación de las características, funcionalidad y estructura de Sistemas Distribuidos, Redes de Computadoras e Internet; y (ii) Capacidad para diseñar sistemas, aplicaciones y servicios basados en tecnologías de red, incluyendo Internet, web, comercio electrónico, multimedia, servicios interactivos e informática móvil. Además, también contribuye a las competencias transversales que incluyen (i) comprensión e integración, (ii) aplicación y pensamiento práctico, (iii) hardware específico y (iv) trabajo en grupo y liderazgo. Como curso de especialización en TI, tiene como objetivo desarrollar una comprensión profunda sobre el funcionamiento y la gestión de dispositivos comerciales de red de área local (LAN), incluidas las tecnologías más ampliamente implementadas (IEEE 802.3 y sus variantes, IEEE 802.11 y sus variantes) y protocolos (VLAN, STP/RSTP, etc.), incluidos los problemas relacionados con la seguridad. También se proporciona una breve descripción de las tecnologías de acceso, y otros estándares de LAN.

La asignatura DCLAN se desarrolla en el sexto semestre de la titulación ( $3^{\circ}$ curso), durante el período que va de febrero a mayo, y está dotada de 4,5 créditos ECTS. Estos 4,5 créditos se dividen en créditos de teoría (3 ECTS) y créditos de prácticas (1,5 ECTS). Las clases teóricas se llevan a cabo todas las semanas como una única sesión de 2 horas. Durante estas sesiones, el profesor imparte clases magistrales a los estudiantes usando diferentes recursos como diapositivas, vídeos y pizarra. Estas clases magistrales se intercalan frecuentemente con cuestionarios y pruebas breves para los estudiantes. Las sesiones de laboratorio también tienen lugar una vez por semana. Durante las sesiones de laboratorio, los estudiantes tienen contacto directo con hardware de red, como puntos de acceso e interruptores de alto rendimiento, para realizar tareas de administración. Además, los estudiantes también realizan simulaciones de redes para conocer el desempeño de las diferentes tecnologías de red bajo diferentes cargas, entre otros objetivos. En cuanto a la evaluación, se definen un total de cinco actividades de evaluación, dos de ellas relacionadas con los contenidos de las clases teóricas, y tres relacionadas con los contenidos de las sesiones de laboratorio. Los detalles sobre estos actividades de evaluación se presentan en la tabla 1.

Cabe señalar que la repetición de los exámenes correspondientes a los dos exámenes escritos, relacionados con la teoría, también está prevista para mediados de junio, una vez finalizadas las clases. 
Tabla 1: Actos de evaluación (planificación inicial).

\begin{tabular}{|c|c|c|c|}
\hline Clasificación & Número/tipo & $\begin{array}{c}\text { Peso } \\
\mathbf{( \% )}\end{array}$ & Fechas \\
\hline \hline Asociados a la teoría & 2 pruebas escritas & $60 \%$ & Abril / Junio \\
\hline $\begin{array}{c}\text { Asociados a las prácticas } \\
\text { de laboratorio }\end{array}$ & 2 exámenes tipo test & $20 \%$ & $\begin{array}{c}\text { Marzo / } \\
\text { Mayo }\end{array}$ \\
\cline { 2 - 4 } & 1 trabajo en grupo & $20 \%$ & Mayo \\
\hline
\end{tabular}

Tabla 2: Eventos de evaluación (planificación final). Los cambios importantes en comparación con la planificación inicial se destacan en negrita.

\begin{tabular}{|c|c|c|c|}
\hline Clasificación & Número/tipo & $\begin{array}{c}\text { Peso } \\
\mathbf{( \% )}\end{array}$ & Fechas \\
\hline \hline Asociados a la teoría & 2 exámenes online & $60 \%$ & Abril / Junio \\
\hline \multirow{2}{*}{$\begin{array}{c}\text { Asociados a las prácticas } \\
\text { de laboratorio }\end{array}$} & $\begin{array}{c}\text { Entregable de } \\
\text { prácticas }\end{array}$ & $\mathbf{1 0 \%}$ & Marzo \\
\cline { 2 - 4 } & $\begin{array}{c}\text { Entregable del trabajo } \\
\text { en grupo }\end{array}$ & $\mathbf{1 5 \%}$ & Mayo \\
\cline { 2 - 4 } & $\begin{array}{c}\text { Test online relativo } \\
\text { al trabajo }\end{array}$ & $\mathbf{1 5 \%}$ & Mayo \\
\hline
\end{tabular}

\section{Cambios docentes realizados}

A mediados de marzo de 2020, la crisis del COVID-19 que afectó a España y a muchos otros países del mundo, ha provocado cambios bruscos en todos los ámbitos académicos, provocando que las clases universitarias pasaran forzosamente a ser impartidas mediante una docencia a distancia, con el curso ya comenzado. Es importante destacar que, evidentemente, la docencia estaba planificada y diseñada siguiendo los enfoques reglados de la docencia presencial tradicional, esto es, con presencia física de alumnos y profesorado, evaluación presencial, etc. En la UPV, el procedimiento adoptado consistió en adecuar todas las guías docentes de las asignaturas para incorporar las acciones necesarias para facilitar este repentino cambio. En realidad, este proceso se llevó a cabo en dos fases; una primera modificación de urgencia que describe los principales cambios que tuvieron lugar durante el período de confinamiento estricto (incluyendo demasiados supuestos), y una segunda modificación, ya definitiva, cuando, más tarde, quedó claro que volver a clases no era una opción, y que todos los eventos de evaluación deberían basarse en plataformas virtuales. En aras de simplicidad, simplemente procedemos a detallar la planificación final de los eventos de evaluación, como se muestra en la tabla 2.

Como se puede apreciar, estos cambios afectaron más a las actividades relacionadas con el laboratorio. Al cerrar las instalaciones de la universidad, los estudiantes no podían acceder a las sesiones de laboratorio. En el caso particular de la asignatura DCLAN, la mayoría de las sesiones de laboratorio requieren manejar hardware real, solo disponible en el laboratorio. Esto implico la buscada de recursos software que suplieran, de algún modo esta carencia, así como una planificación alternativa de estas nuevas actividades. Por lo tanto, para la primera mitad de las sesiones de laboratorio, un esquema de evaluación basado en un entregable, elegido al azar entre las diferentes sesiones de laboratorio ya completadas, reemplazó al primer examen tipo prueba. Para el segundo bloque, que fue cancelado, la alternativa propuesta consistió en extender el proyecto y su procedimiento de evaluación. Así, de manera similar a la planificación inicial, el proyecto grupal tenía una parte 


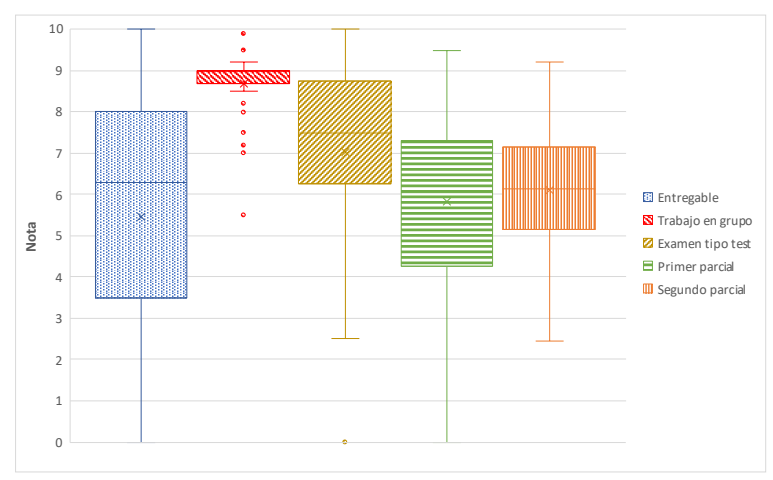

Fig. 1: Resultados de las notas obtenidas en los diferentes actos de evaluación.

entregable, cuyo peso se incrementó del $10 \%$ al $15 \%$ en la nueva planificación. Sin embargo, la puntuación restante para completar el $20 \%$ definido en la planificación inicial, la cual dependía inicialmente de una entrevista/cuestiones al estudiante en el laboratorio, fue reemplazada por un test a través del campus virtual en la planificación final. De esta forma, la puntuación del proyecto se incrementó del $20 \%$ inicial al $15 \%+15 \%=30 \%$ final, lo que nos permitió mantener el peso total relacionado con las actividades del laboratorio en un $40 \%$, tal como se observa en la tabla 2 .

\section{Resultados de evaluación y percepción del alumnado}

En este apartado se procede a presentar y discutir los resultados de la evaluación obtenidos, comparándolos con otras evaluaciones similares realizadas en años anteriores, cuando corresponda. Adicionalmente, presentamos los resultados de percepción de los estudiantes sobre el grado de representatividad de las notas obtenidas.

En lo referente a las notas obtenidas por los estudiantes en los diferentes actos de evaluación, la Figura 1 muestra que las notas del entregable de prácticas son las que se distribuyen más ampliamente por el rango de notas. Destacar que exigía un esfuerzo individual ya que el entregable era un informe personal de cada uno, que exigía una interpretación con sus propias palabras y en base a sus propios resultados recabados durante las sesiones de prácticas, por lo que se considera que representa de manera correcta la distribución de conocimientos en ese apartado. El trabajo en grupo muestra la menor variabilidad de notas. Esto se debe a que es un trabajo guiado, con posibilidad de tutorización para validación del mismo, y con un amplio margen de tiempo para su ejecución. Por el contrario, en el examen tipo test correspondiente a ese trabajo, ahora sí individual y con tiempo acotado, se verifica que la distribución de notas es bastante más amplia.

Con respecto a los cursos anteriores, solo las notas de ambos parciales son directamente comparables, al haber cambios en las otras actividades de evaluación derivados del COVID-19. Siendo así, las Figuras 2 y 3 muestran que claramente hay una tendencia al alza en las notas para el curso 2019-20, tanto la media como la mediana superan a todos los cursos anteriores, a pesar de que las diferencias de rango para el primer parcial son menores. De hecho, en ese primer parcial, la mediana supera incluso el tercer cuartil de todos los cursos anteriores, lo cual hace hincapié en las diferencias significativas entre las distribuciones de notas. Para el segundo parcial las diferencias son más marcadas para la desviación de notas, teniéndose la distancia inter-cuartil más reducida que nunca, siendo las diferencias más marcadas para las notas por debajo de la mediana. En resu- 


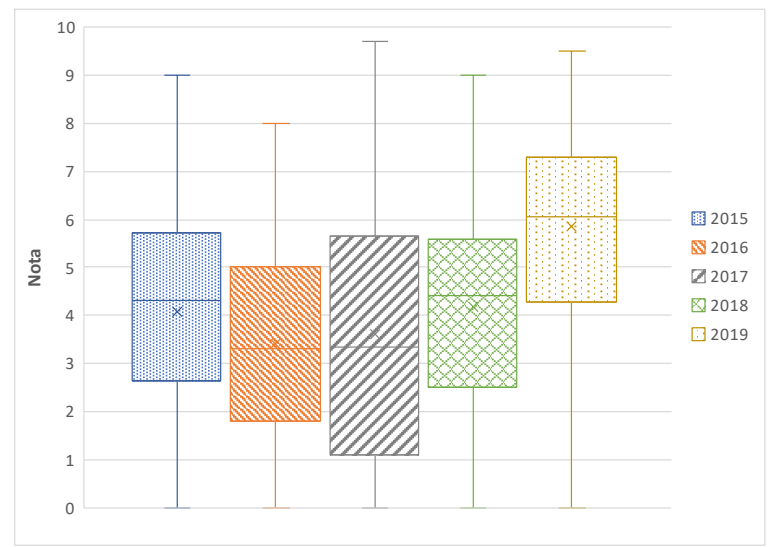

Fig. 2: Comparativa de las notas obtenidas en el primer parcial para los distintos cursos.

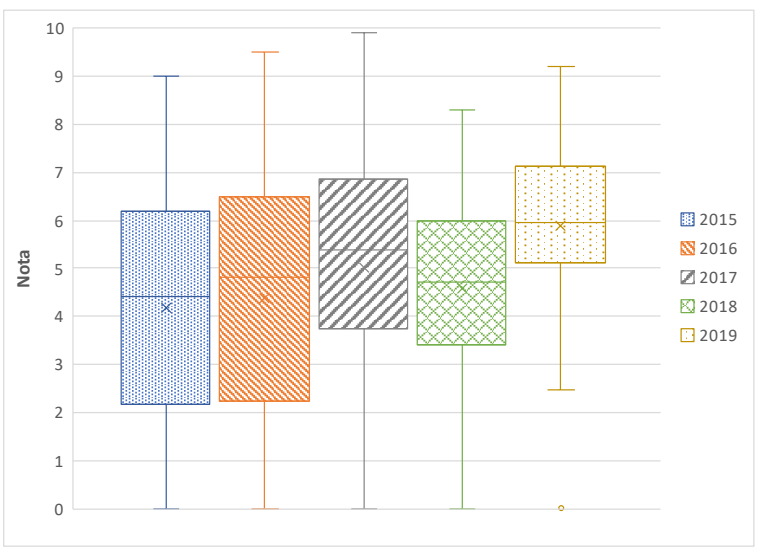

Fig. 3: Comparativa de las notas obtenidas en el segundo parcial para los distintos cursos.

men, en ambos actos de evaluación se verifica una tendencia al alza de las notas, siendo esta más marcada para los valores altos en el primer parcial, y para los valores bajos en el segundo parcial.

El efecto global en los resultados finales se muestran en la Figura 4, donde se puede ver claramente que el curso 2019 supera a todos los anteriores en términos de: media, mediana, cuartiles Q1 y Q3, así como del valor inferior del rango de la caja. En la tabla 3 se desglosan los valores concretos de las principales estadísticas para los diferentes cursos. Se comprueba como la media es la más alta y la desviación estándar es la más reducida del histórico; además, con un 91,8\%, la tasa de aprobados es la más alta de la serie histórica registrada. Destacar también que el error muestral para estos resultados se considera prácticamente nulo ya que la casi totalidad de los estudiantes ha participado en los diferentes actos de evaluación que aquí se detallan.

En lo que respecta a la percepción del alumnado respecto a la efectividad de los diferentes actividades de evaluación para garantizar la ausencia de copia/plagio por parte del alumnado, se ha realizado una encuesta donde se pedía al alumno que valorase de 1 a 10, siendo 10 el máximo de efectividad, y 1 el mínimo de efectividad, su experiencia y opinión para cada acto de evaluación atendiendo a las características de cada uno de esos actos, tal y como se detalla en la tabla 4. La tasa de participación en esta encuesta ha sido del $35 \%$. 
Tabla 3: Estadísticas referentes a la nota final en los distintos cursos.

\begin{tabular}{|c|c|c|c|}
\hline $\begin{array}{c}\text { Acto } \\
\text { evaluación }\end{array}$ & Média & $\begin{array}{c}\text { Desviación } \\
\text { estándar }\end{array}$ & $\begin{array}{c}\text { Tasa de } \\
\text { aprobados } \\
\text { (\%) }\end{array}$ \\
\hline \hline $2015-16$ & 5,37 & 1,52 & $78,9 \%$ \\
\hline $2016-17$ & 4,84 & 1,79 & $67,3 \%$ \\
\hline $2017-18$ & 5,08 & 1,92 & $71,4 \%$ \\
\hline $2018-19$ & 5,35 & 1,46 & $77,9 \%$ \\
\hline $2019-20$ & 6,48 & 1,33 & $91,8 \%$ \\
\hline
\end{tabular}

Tabla 4: Características de los diferentes actos de evaluación

\begin{tabular}{|c|c|c|}
\hline $\begin{array}{c}\text { Acto } \\
\text { evaluación }\end{array}$ & Método usado & Deficiencias encontradas \\
\hline $\begin{array}{l}\text { Entregable } \\
\text { individual } \\
\text { relativo a las } \\
\text { sesiones de } \\
\text { prácticas }\end{array}$ & $\begin{array}{l}\text { Entregable aleatorio para cada } \\
\text { alumno, y resumen comentado } \\
\text { personal. }\end{array}$ & $\begin{array}{c}\text { Al haber solo } 4 \text { sesiones } \\
\text { diferentes y un plazo } \\
\text { alargado, es sencillo } \\
\text { encontrar compañeros con el } \\
\text { mismo trabajo. }\end{array}$ \\
\hline $\begin{array}{l}\text { Trabajo } \\
\text { Packet Tracer } \\
\text { (en pareja) }\end{array}$ & $\begin{array}{c}\text { Escenario de partida con watermark } \\
\text { distinto para cada grupo para evitar la } \\
\text { copia. }\end{array}$ & $\begin{array}{l}\text { Al haber un trabajo único y } \\
\text { un plazo alargado, es posible } \\
\text { intercambiar información } \\
\text { entre compañeros. }\end{array}$ \\
\hline $\begin{array}{l}\text { Examen tipo } \\
\text { test sobre } \\
\text { trabajo }\end{array}$ & $\begin{array}{c}\text { Preguntas aleatórias de un pool } 4 \\
\text { veces superior al tamaño del examen. } \\
\text { Una vez respondida una pregunta se } \\
\text { pasa a la siguiente sin hay posibilidad } \\
\text { de volver atrás. Tiempo acotado a } 4 \\
\text { minutos por pregunta en media. }\end{array}$ & $\begin{array}{l}\text { Al ser un examen tipo test, } \\
\text { es posible a los alumnos más } \\
\text { rápidos compartir las } \\
\text { respuestas con los más } \\
\text { rezagados. }\end{array}$ \\
\hline Primer parcial & $\begin{array}{l}\text { Pool de preguntas aproximadamente } 6 \\
\text { veces superior al tamaño del examen } \\
\text { (200.000 posibles exámenes distintos). } \\
\text { Tiempo acotado a } 10 \text { minutos por } \\
\text { pregunta en media. }\end{array}$ & $\begin{array}{l}\text { Hay tiempo suficiente para } \\
\text { encontrar compañeros con } \\
\text { preguntas similares. Se han } \\
\text { detectado plagios. }\end{array}$ \\
\hline $\begin{array}{l}\text { Segundo } \\
\text { parcial }\end{array}$ & $\begin{array}{l}\text { Pool de preguntas aproximadamente } 5 \\
\text { veces superior al tamaño del examen ( } 7 \\
\text { millones posibles examenes distintos). } \\
\text { Preguntas orientadas al análisis, } \\
\text { comprensión e interpretación personal. }\end{array}$ & $\begin{array}{l}\text { Hay tiempo suficiente para } \\
\text { encontrar compañeros con } \\
\text { preguntas similares. Se han } \\
\text { detectado plagios. }\end{array}$ \\
\hline
\end{tabular}




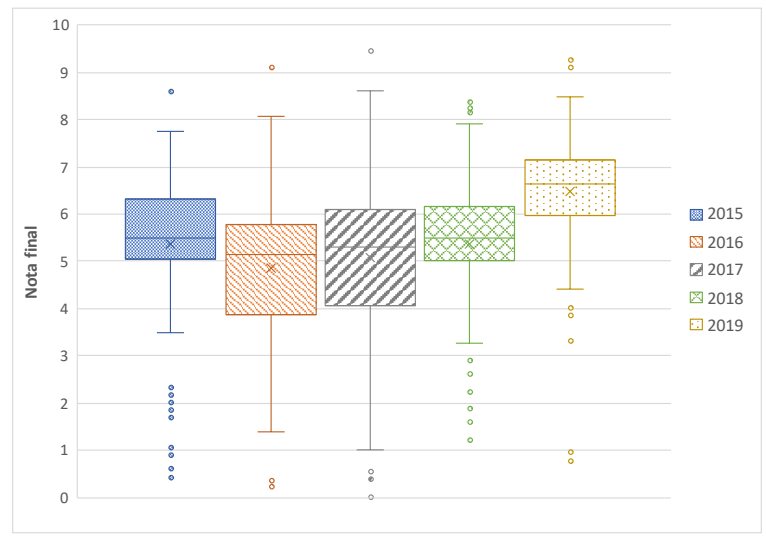

Fig. 4: Comparativa de las notas finales obtenidas para los distintos cursos.

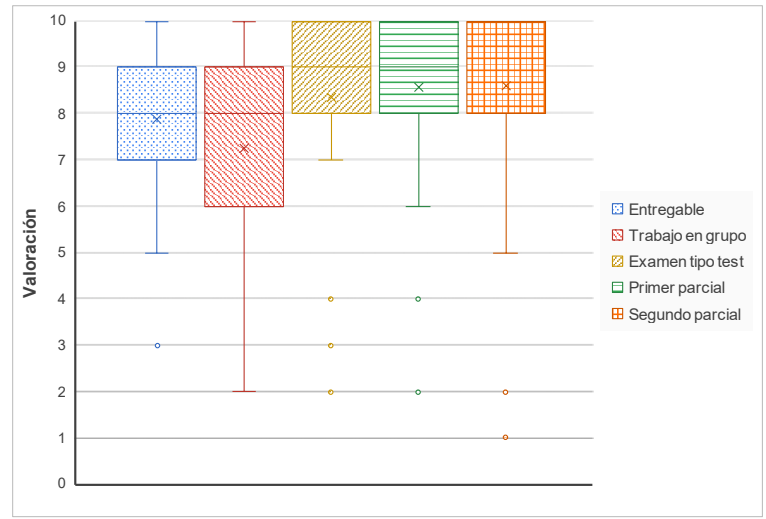

Fig. 5: Resultados de la encuesta al alumnado relativos a la percepción de los actos de evaluación.

En la Figura 5 se muestran los resultados de la encuesta, donde se puede observar que los alumnos, en general, valoran favorablemente las efectividad de las distintas estrategias para evitar plagio en las actividades de evaluación realizadas durante el curso. No obstante, destaca como método mejor valorado el test a través del campus virtual, para medir los conocimientos del trabajo práctico realizado con la herramienta Packet Tracer de Cisco. Creemos que esto se debe principalmente a que, en esta prueba, se combinaban dos factores: aleatoriedad en las preguntas de cada alumno y, más importante, la imposibilidad de volver atrás una vez se había respondido a una pregunta, lo que ciertamente complica poder consensuar las respuestas con el resto de compañeros. Curiosamente, la actividad de evaluación que recibió una valoración más baja ha sido el propio trabajo realizado con Packet Tracer. En este caso, a pesar de que cada grupo tenía un escenario de partida único, establecido mediante marca de agua, es cierto que el trabajo era común para todos, y se disponía de un amplio margen de tiempo para su elaboración, por lo cual era más sencillo obtener y consensuar información de otros compañeros. En este sentido, destacar que dicha posibilidad ya había sido tenida en cuenta, razón por la que se complementó dicho trabajo con el test mediante el campus virtual, permitiendo así que la nota combinada de ambos actos refleje de forma más adecuada la adquisición de las competencias de cada alumno. 


\section{Conclusiones}

El año académico 2019-2020 será recordado en las universidades por los grandes cambios que tuvieron que experimentar la enseñanza tradicional para convertirla en enseñanza a distancia en cuestión de días. Aunque en nuestra institución la mayoría de las asignaturas lograron este objetivo con un éxito notable, recibiendo una amplia aceptación entre los estudiantes, tanto profesores como estudiantes reconocen que algunas partes del proceso no cumplieron con los estándares de calidad esperados. En este trabajo nos centramos en el caso particular de los procedimientos y resultados de evaluación. Encontramos que, a pesar de los esfuerzos para equilibrar el procedimiento de evaluación al de años anteriores en términos tanto de contenidos como de adquisición de competencias, las puntuaciones obtenidas fueron significativamente más elevadas que en el histórico registrado de la asignatura. Así, a pesar de los grandes esfuerzos realizados por el profesorado para evitarlo, las evaluaciones a distancia han sido objeto de problemas de plagio entre los estudiantes. Se tienen además sospechas fundadas de que parte del alumnado se coordinaban para compartir sus respuestas durante las actividades de evaluación, ya que se han identificado pruebas fehacientes en exámenes prácticamente idénticos. Preguntados a los estudiantes sobre su percepción de equidad/adecuación de las puntuaciones obtenidas en las diferentes actividades de evaluación, los resultados de la encuesta realizada al alumnado mostraron grandes disparidades en sus opiniones, al tiempo que evidenciaron que, de hecho, algunas de las evaluaciones se percibieron como poco fiables en términos de resultados.

Como trabajo futuro, planeamos comparar estos resultados con los que se llevarán a cabo durante el año académico 2020-2021, donde se ha adoptado igualmente una enseñanza a distancia, donde las evaluaciones programadas, sin embargo, son presenciales. Además, este trabajo puede ser la base de nuevos estudios de investigación sobre: (i) sistemas de evaluación que sean simples y eficaces cuando se deba realizar una evaluación remota; (ii) soluciones avanzadas contra el plagio; (iii) evaluación de las diferencias del proceso de enseñanza/aprendizaje reales entre estudiantes a distancia y estudiantes presenciales durante el curso de una misma materia, profesor y período de tiempo.

\section{Referencias bibliográficas}

Alruwais, Nuha, Gary Wills y Mike Wald (2018). "Advantages and challenges of using e-assessment". En: International Journal of Information and Education Technology 8.1, págs. 34-37.

Bao, Wei (2020). "COVID-19 and online teaching in higher education: A case study of Peking University". En: Human Behavior and Emerging Technologies 2.2, págs. 113-115.

Brown, Gavin TL (2020). "Schooling Beyond COVID-19: An Unevenly Distributed Future". En: Frontiers in Education. Vol. 5. Frontiers, pág. 82.

Cantabella, Magdalena y col. (2020). "Evaluation of parallel programming teaching methodologies: On-campus versus online methodologies". En: Computer Applications in Engineering Education 28.2, págs. 229-238.

Ferdig, Richard E y col. (2020). "Teaching, technology, and teacher education during the covid-19 pandemic: Stories from the field". En: Waynesville, NC, USA: Association for the Advancement of Computing in Education (AACE). 
Kearns, Lorna R (2012). "Student assessment in online learning: Challenges and effective practices". En: Journal of Online Learning and Teaching 8.3, pág. 198.

Mishra, Lokanath, Tushar Gupta y Abha Shree (2020). "Online Teaching-Learning in Higher Education during Lockdown Period of COVID-19 Pandemic". En: International Journal of Educational Research Open, pág. 100012.

Nicola, Maria y col. (2020). "The socio-economic implications of the coronavirus pandemic (COVID19): A review". En: International journal of surgery (London, England) 78, pág. 185.

Nuere, Silvia y Laura de Miguel (2020). "The Digital/Technological Connection with COVID-19: An Unprecedented Challenge in University Teaching". En: Technology, Knowledge and Learning, págs. 1-13.

Odriozola-González, Paula y col. (2020). "Psychological effects of the COVID-19 outbreak and lockdown among students and workers of a Spanish university". En: Psychiatry Research, pág. 113108.

Rapanta, Chrysi y col. (2020). "Online university teaching during and after the Covid-19 crisis: Refocusing teacher presence and learning activity". En: Postdigital Science and Education, págs. 1-23.

Sahu, Pradeep (2020). "Closure of universities due to Coronavirus Disease 2019 (COVID-19): impact on education and mental health of students and academic staff". En: Cureus 12.4.

Schleicher, Andreas (2020). The impact of COVID-19 on education: insights from Education at a Glance 2020. OECD, Paris, France.

Timmis, Sue y col. (2016). "Rethinking assessment in a digital age: Opportunities, challenges and risks". En: British Educational Research Journal 42.3, págs. 454-476. 